Hardy Ramanujan Journal

Vol. 32 (2009) 38-53

\title{
Finite expressions for higher derivatives of the Dirichlet $L$-function and the Deninger $R$-function
}

\author{
K. Chakraborty, S. Kanemitsu, and T. Kuzumaki \\ Dedicated to Professor Dr. Harald Niederreiter on his sixtieth birthday \\ with great respect and friendship
}

\begin{abstract}
In this paper we are concerned with the equivalence of the finite expression for the derivative of the $R$-function at rational arguments and the Kronecker limit formula in the spirit of our former study on the Gauss formula for the digamma function and the Dirichlet class number formula. In the present case, the Gauss formula and the Dirichlet class number formula are to be replaced by its analogue for the $R$-function and by the Kronecker limit formula or rather a closed form for the derivative of the Dirichlet $L$ function, respectively. To make a systematic study on the $R_{k}$-functions, we appeal to our recent method of using the Lipschitz-Lerch transcendent in which there is the vector space structure built-in of those special functions that we need. By way of the generalized Euler constants, we deduce the closed formula given in Theorem 1.
\end{abstract}

2000 Mathematics Subject Classification: 11F66, 11M26, 11M41.

\section{Introduction and statement of results}

The closed formula for the first derivative of the Dirichlet $L$-function at $s=1$ is essential in expressing the Kronecker limit formula in another form to arrive at the Lerch-Selberg-Chowla formula [Ler2], [CSel], [SelC] (for its intereting history, cf. [Kur] and [Kum]). In the case of imaginary quadratic fields, the formula has been known from the time of Dirichlet, while in the case of real quadratic fields, the key function $R(x)$ came to be known only by the work of Gut $[\mathrm{Gu}]$, which was subsequently improved in a systematic way by Deninger [Den]. Among other things, in analogy to the Gauss formula, for $(\log \Gamma)^{\prime}$ (cf. e. g. [Vista, (2.58), p. 47]), Deninger proved [Den] (2.2), which is most relevant to us. 
Our objective of this paper is two-fold. In $\S 1$, we shall obtain closed form for the derivatives of the Dirichlet $L$-function at $s=1$ (Theorem 1), while in $\S 2$, we shall give an equivalent formulation of the derivative of the $R$-function in terms of the closed form for the derivative of the Dirichlet $L$-function, in the spirit of [HKT] (cf. also [Vista, Chapter 8]). The method in $\S 1$ hinges on the comparison of the Taylor expansion at $s=0$ and the Laurent expansion at $s=1$ of (1.6), which in turn is an elaboration of our recent method $[\mathrm{LCK}]$, which we shall now explain.

Let

$$
\zeta(s, a)=\sum_{n=0}^{\infty} \frac{1}{(n+a)^{s}}, \quad \sigma=\operatorname{Re} s>1
$$

denote the Hurwitz zeta-function, which is continued to a meromorphic function over the whole plane with a unique simple pole at $s=1$. The special case $a=1$ of $\zeta(s, a)$ is the well-known Riemann zeta-function

$$
\zeta(s)=\zeta(s, 1)
$$

whose treatment will be included in that of the Hurwitz zeta-function, and we will freely use the results on the Riemann zeta-function. The Taylor expansion of the Hurwitz zeta-function in the second variable was obtained as early as $1922 / 23$ by Wilton [Will, (1), p. 90]

$$
\sum_{n=0}^{\infty} \frac{(s)_{n}}{n !} \zeta(s+n, a) z^{n}=\zeta(s, a-z), \quad|z|<|a|
$$

valid for all $s \neq 1$.

More generally, let $L(x, s, a)$ denote the Lipschitz-Lerch transcendent (often called the Hurwitz- Lerch zeta-function) defined by

$$
L(x, s, a)=\sum_{n=0}^{\infty} \frac{e^{2 \pi i n x}}{(n+a)^{s}}, \quad \sigma=\operatorname{Re} s>1,
$$

which was first studied by Lipschitz [Lip] and Lerch [Ler1] independently. Here the paprameters are to the effect that $x$ expresses the signature and $a$ the perturbation. We refer to [LG] for a systematic treatment of the Lipschitz-Lerch transcendent, where it is referred to as the Lerch zetafunction. For general references on special functions, we refer to [Erd], [SC] and [Vista]. 
Klusch [Klu] obtained the Taylor expansion of $L(x, s, a+z)$ at $z=0$

$$
\sum_{n=0}^{\infty}(-1)^{n} \frac{(s)_{n}}{n !} L(x, s+n, a) z^{n}=L(x, s, a+z), \quad|z|<|a| .
$$

The proof of (1.4) depends on the differential-difference equation

$$
\frac{\partial}{\partial a} L(x, s, a)=-s L(x, s+1, a) .
$$

We also refer the reader to Srivastava-Choi [SC, p. 125] for (1.4) and (1.5).

However, if $x \notin \mathbb{Z}$, then $L(x, s, a)$ is continued to an integral function and we do not have the Laurent expansion to be compared with (1.5), while if $x \in \mathbb{Z}$, then $L(x, s, a)$ reduces to the Hurwitz zeta-function. Hence we may start from the Hurwitz zeta-function. We shall appeal to the following functional equation of it with the perturbation parameter being rational.

Proposition 1. ([Ap, Theorem 128, p. 261], [SC, (8), p. 90]). For all $s \neq 0,1$, we have

$$
\zeta\left(1-s, \frac{p}{q}\right)=\frac{2 \Gamma(s)}{(2 \pi q)^{s}} \sum_{a=1}^{q} \cos \left(\frac{\pi s}{2}-\frac{2 \pi a p}{q}\right) \zeta\left(s, \frac{a}{q}\right) .
$$

One can also derive Proposition 1 from the following lemma (cf. [LCK]).

Lemma 1. For $1<q \in \mathbb{Z}$ and $q>p \in \mathbb{N}$, we have the identity

$$
\begin{aligned}
& q^{-s} \sum_{a=1}^{q} e^{2 \pi i \frac{a}{q} p} \zeta\left(s, \frac{a}{q}\right)\left(=l_{s}\left(\frac{p}{q}\right)=\right) \\
& \quad=i \frac{\Gamma(1-s)}{(2 \pi)^{1-s}}\left\{e^{-\frac{\pi i s}{2}} \zeta\left(1-s, \frac{p}{q}\right)-e^{\frac{\pi i s}{2}} \zeta\left(1-s, 1-\frac{p}{q}\right)\right\},
\end{aligned}
$$

where, for $s=0$ or $s=1$, the identity is to mean the limit as $s \rightarrow 0$ or $s \rightarrow 1$.

It is easily seen that Lemma 1 is a special case of

Proposition 2. For $1<q \in \mathbb{Z}$ and $0<x<1$, Re $a>0$, we have the identity

$$
\begin{aligned}
& q^{-s} \sum_{j=0}^{q-1} L\left(q x, s, \frac{a+j}{q}\right) e^{2 \pi i j x} \\
& =i \frac{\Gamma(1-s)}{(2 \pi)^{1-s}}\left\{e^{\frac{(1-s) \pi i}{2}} L(-a, 1-s, x)-e^{-\frac{(1-s) \pi i}{2}} L(-a, 1-s, 1-x)\right\}
\end{aligned}
$$


except at the singularities of $L$, in which case, the identity is to mean the limit.

It has been shown in [LCK], that (1.6) and its counterpart for the polylogarithm function entail not only all the previous results but also new nontrivial corollaries.

Let us recall [Vista, p. 54] that the Laurent expansion of $\zeta(s, x)$ at $s=1$ is given by (cf. [Vista, Corollary 3.3])

$$
\zeta(s, x)=\frac{1}{s-1}+\sum_{n=0}^{\infty} \frac{(-1)^{n} \gamma_{n}(x)}{n !}(s-1)^{n}, \quad s \rightarrow 1,
$$

where $\gamma_{0}(x)=-\psi(x)$ is the Euler digamma function to be introduced in (1.20), or

$$
\zeta(s+1, x)=\frac{1}{s}+\sum_{n=0}^{\infty} \frac{(-1)^{n} \gamma_{n}(x)}{n !} s^{n}, \quad s \rightarrow 0,
$$

where $\gamma_{k}(a)$ denotes the $k$-th Laurent coefficient and expression for this can be found in [Vista, Chapter 3, Page 60-61].

Now we denote the Taylor coefficients (up to a constant multiple) of $\zeta(s, x)$ at $s=0$ by

$$
R_{n}(x)=\left.(-1)^{n-1} \frac{\partial^{n}}{\partial s^{n}} \zeta(s, x)\right|_{s=0},
$$

or

$$
\zeta(s, x)=-\sum_{n=0}^{\infty} \frac{(-1)^{n} R_{n}(x)}{n !} s^{n} .
$$

Differentiating (1.9) with respect to $x$, we obtain

$$
\frac{\partial}{\partial x} \zeta(s, x)=-\sum_{n=0}^{\infty} \frac{(-1)^{n} R_{n}^{\prime}(x)}{n !} s^{n} .
$$

Substituting (1.7) and (1.10) in the special case of (1.5)

$$
\frac{\partial}{\partial a} \zeta(x, s)=-s \zeta(x, s+1),
$$

and comparing the coefficients, we deduce the following important relation. 


\section{Lemma 2.}

$$
R_{n}^{\prime}(x)=-n \gamma_{n-1}(x) .
$$

To state the main result, we need to introduce some notations. Let $p, q \in \mathbb{N}$ with $p<q$ and $(p, q)=1$. Let

$$
-K_{l}\left(\frac{p}{q}, r\right)=\sum_{m=0}^{l}\left(\begin{array}{c}
l \\
m
\end{array}\right) R_{l-m}\left(\frac{r}{q}\right)\left(\frac{\pi}{2}\right)^{m} T_{m}\left(\frac{r p}{q}\right),
$$

where

$$
T_{m}(x)=\left\{\begin{array}{cc}
(-1)^{m / 2} \cos (2 \pi x), & m \text { even }, \\
(-1)^{(m+1) / 2} \sin (2 \pi x), & m \text { odd }
\end{array}\right.
$$

and

$$
\delta_{l}=\sum_{m=0}^{l}(-1)^{m}\left(\begin{array}{c}
l \\
m
\end{array}\right) \Gamma^{(m)}(1)(\log 2 \pi q)^{l-m} .
$$

We now state and prove the main result of this section.

Theorem 1.

$$
R_{n}^{\prime}\left(\frac{p}{q}\right)=2(-1)^{n} \sum_{l=0}^{n}\left(\begin{array}{l}
n \\
l
\end{array}\right) \delta_{n-l} \sum_{r=1}^{q} K_{l}\left(\frac{p}{q}, r\right) .
$$

Proof. In view of (1.7) and Lemma 2, the right-hand side of (1.6) is

$$
\zeta\left(1-s, \frac{p}{q}\right)=-\frac{1}{s}-\frac{1}{s} \sum_{n=1}^{\infty} \frac{1}{n !} R_{n}^{\prime}\left(\frac{p}{q}\right) s^{n} .
$$

To find the expansion of the right-hand side, we note that

$$
\cos \left(\frac{\pi s}{2}-\frac{2 \pi r p}{q}\right)=\sum_{m=0}^{\infty} \frac{1}{m !}\left(\frac{\pi}{2}\right)^{m} T_{m}\left(\frac{r p}{q}\right) s^{m},
$$

where

$$
T_{m}(x)=i^{m} \frac{1}{2}\left(e^{-2 \pi i x}+(-1)^{m} e^{2 \pi i x}\right)
$$

is as given by (1.13). Also it is immediate to see from (1.14) that

$$
\frac{2 \Gamma(s)}{(2 \pi q)^{s}}=\frac{2}{s} \sum_{m=0}^{\infty} \frac{(-1)^{l}}{l !} \delta_{l} s^{l} .
$$


Multiplying (1.17) and (1.18), we see that the coefficient of $s^{n}$ is -1 times the right-hand side of (1.15). Comparing it with (1.16) completes the proof.

Corollary 1. Formula (1.15) in the case $n=1$ amounts to the Gauss formula

$$
\psi\left(\frac{p}{q}\right)=-\gamma-\log q-\frac{\pi}{2} \cot \frac{p}{q} \pi+\sum_{k=1}^{q-1} \cos \frac{2 p k}{q} \pi \log 2 \sin \frac{k}{q} \pi,
$$

where

$$
\psi(z)=(\log \Gamma)^{\prime}(z)=\frac{\Gamma^{\prime}}{\Gamma}(z)
$$

is the Euler digamma function (cf. e. g. [Vista, Chapter 5]) and $\gamma=$ $-\Gamma^{\prime}(1)=-\psi(1)$ is the Euler constant, and in the case $n=2$ it amounts to the Deninger formula

$$
\begin{aligned}
& R^{\prime}\left(\frac{p}{q}\right)=-\gamma_{1}-2(\gamma+\log 2 \pi q)\left(\gamma+\psi\left(\frac{p}{q}\right)\right) \\
& +2 \sum_{a=1}^{q-1}\left(\cos \frac{2 \pi a p}{q}-1\right) R\left(\frac{a}{q}\right)-2 \pi \sum_{a=1}^{q-1} \log \Gamma\left(\frac{a}{q}\right) \sin \frac{2 \pi a p}{q}
\end{aligned}
$$

where $\gamma_{1}=\gamma_{1}(1)$ is the first generalized Euler constant.

Proof. We prove (1.21) which simultaneously will prove (1.19) too.

One has

$$
\delta_{0}=1, \quad \delta_{1}=\log 2 \pi q+\gamma .
$$

Now noting that

$$
\delta_{2}=(\log 2 \pi q)^{2}+2 \gamma(\log 2 \pi q)+\Gamma^{\prime \prime}(1),
$$

and that $\Gamma^{\prime \prime}(1)=\frac{\pi^{2}}{6}-\gamma^{2}$, we obtain

$$
\delta_{2}=(\log 2 \pi q)^{2}+2 \gamma(\log 2 \pi q)+\frac{\pi^{2}}{6}-\gamma^{2} .
$$

We also have

$$
\sum_{r=1}^{q} K_{0}\left(\frac{p}{q}, r\right)=-\frac{1}{2}
$$


on using

$$
\sum_{r=1}^{q-1} \cos \frac{2 \pi r p}{q}=-1
$$

Next,

$$
\sum_{r=1}^{q} K_{1}\left(\frac{p}{q}, r\right)=\frac{1}{2} \sum_{r=1}^{q-1} \cos \frac{2 \pi r p}{q}\left(\log \sin \frac{\pi r}{q}\right)-\frac{\pi}{4} \cot \frac{\pi p}{q}-\frac{1}{2} \log \pi,
$$

which takes the shape (by (1.19)),

$$
\sum_{r=1}^{q} K_{1}\left(\frac{p}{q}, r\right)=\frac{1}{2}\left(\psi\left(\frac{p}{q}\right)+\gamma\right)+\frac{1}{2} \log 2 \pi q .
$$

Also we have,

$$
\sum_{r=1}^{q} K_{2}\left(\frac{p}{q}, r\right)=\sum_{r=1}^{q-1} R\left(\frac{r}{q}\right) \cos \frac{2 \pi r p}{q}+R(1)-\pi \sum_{r=1}^{q-1} \log \Gamma\left(\frac{r}{q}\right) \sin \frac{2 \pi r p}{q}+\frac{\pi^{2}}{8} .
$$

Now using

$$
R(1)=R(2)=-\zeta^{\prime \prime}(0)=\frac{1}{2}\left(\log ^{2} 2 \pi+\frac{\pi^{2}}{12}-\gamma^{2}-\gamma_{1}\right)
$$

we get

$$
\begin{aligned}
\sum_{r=1}^{q} K_{2}\left(\frac{p}{q}, r\right) & =\sum_{r=1}^{q-1} R\left(\frac{r}{q}\right) \cos \frac{2 \pi r p}{q}-\pi \sum_{r=1}^{q-1} \log \Gamma\left(\frac{r}{q}\right) \sin \frac{2 \pi r p}{q} \\
& +\frac{1}{2}\left(\log ^{2} 2 \pi-\gamma^{2}-\gamma_{1}\right)+\frac{\pi^{2}}{12}
\end{aligned}
$$

Finally substituting formulas (1.23)-(1.27) in

$$
R^{\prime}\left(\frac{p}{q}\right)=2\left(\delta_{2} \sum_{r=1}^{q} K_{0}\left(\frac{p}{q}, r\right)+2 \delta_{1} \sum_{r=1}^{q} K_{1}\left(\frac{p}{q}, r\right)+\delta_{0} \sum_{r=1}^{q} K_{2}\left(\frac{p}{q}, r\right)\right)
$$

we conclude (2.2) below, which amounts to (1.21) in view of the distribution property (2.3)(cf. $\S 2$ below). This completes the proof.

Remark 1. The special case $p=q$ of (1.21) is meaningful and amounts to $R^{\prime}(1)=-\gamma_{1}$, which was derived by Deninger [Den, p. 175, 2.3.4]). However, (1.19) for $p=q$ is meaningful only in the sense of the limit

$$
\lim _{p \rightarrow q} \frac{q}{p}\left(\psi\left(\frac{p}{q}+\gamma\right)\right)=-\frac{1}{2}-\log q .
$$




\section{Statement of the equivalence theorem}

Let $R(x)=R_{2}(x)$ denote the Deninger function (cf. Lemma 2 above and [Vista, (5.36), p. 92]) defined for $x>0$ by

$$
R(x)=-\zeta^{\prime \prime}(0, x)=-\left.\frac{\partial^{2}}{\partial s^{2}} \zeta(s, x)\right|_{s=0} .
$$

We rewrite (1.21), for $p, q \in \mathbb{N}$ with $p<q,(p, q)=1$, in the following form

$$
\begin{aligned}
& R^{\prime}\left(\frac{p}{q}\right)=-\gamma_{1}-2(\gamma+\log 2 \pi q)\left(\gamma+\psi\left(\frac{p}{q}\right)\right) \\
& +2 \sum_{a=1}^{q-1} R\left(\frac{a}{q}\right) \cos \frac{2 \pi a p}{q}-2 \pi \sum_{a=1}^{q-1} \log \Gamma\left(\frac{a}{q}\right) \sin \frac{2 \pi a p}{q}-\log q(\log q+2 \log 2 \pi) .
\end{aligned}
$$

We note that relations (1.21) and (2.2) are equivalent under the modified Kubert relation

$$
\sum_{\nu=1}^{q-1} R\left(\frac{\nu}{q}\right)=\frac{1}{2} \log ^{2} q+(\log 2 \pi)(\log q)
$$

for $q \in \mathbb{N}$. This is because the Hurwitz zeta-function satisfies the Kubert relation (distribution property, cf. e.g. [Vista, (3.69), p. 76]), and so the $R$-function and its derivative inherit the same property with minor modification. In fact the derivative satisfies

$$
\sum_{\nu=1}^{q-1} R^{\prime}\left(\frac{\nu}{q}\right)=(q-1) R^{\prime}(1)+q \log ^{2} q+2 \gamma q \log q .
$$

We shall use the following well-known arithmetical functions repeatedly. As always $\mu(n)$ denotes the Möbius function and we shall use its characteristic property

$$
\sum_{d \mid n} \mu(d)= \begin{cases}1, & n=1 \\ 0, & n>1\end{cases}
$$

which is the source of the Möbius inversion formula. We will use (2.5) with $n=(a, q)$ to replace the relatively prime condition $(a, q)=1$ by $\sum_{d \mid(a, q)} \mu(d)$. 
Let $\varphi(q)$ denote the Euler function and we have the expression

$$
\varphi(q)=\sum_{d \mid q} \frac{q}{d} \mu(d) .
$$

Let $c_{q}(\nu)$ denote the Ramanujan sum defined by

$$
c_{q}(\nu)=\sum_{a=1}^{q-1} * e^{2 \pi i a \nu / q},
$$

where $*$ on the summation sign means that the sum is over $a$ with $(a, q)=1$. It is well known that

$$
c_{q}(\nu)=\sum_{d \mid(q, \nu)} d \mu\left(\frac{q}{d}\right) .
$$

For a Dirichlet character modulo $q$, let $G(\mu, \chi)$ denote the generalized Gauss sum defined by

$$
G(\mu, \chi)=\sum_{a=1}^{q-1} \chi(a) e^{2 \pi i \frac{\mu}{q} a}
$$

Then we have

$$
G\left(\nu, \chi_{0}\right)=c_{q}(\nu),
$$

where $\chi_{0}$ indicates the principal character.

For a Dirichlet character $\chi \bmod q$, let

$$
L(s, \chi)=\sum_{n=1}^{\infty} \frac{\chi(n)}{n^{s}}, \quad \sigma=\operatorname{Re} s>1
$$

be the Dirichlet $L$-function. This can be analytically continued to an entire function in the case of $\chi \neq \chi_{0}$ and to a mermorphic function in the case $\chi=\chi_{0}$, and we may speak of the following value in the former case

$$
L^{\prime}(1, \chi)=-\sum_{n=1}^{\infty} \frac{\chi(n) \log n}{n},
$$

which is the fundamental ingredient in the Kronecker limit formula. As in the case of the Dirichlet class number formula, a finite expression for $L^{\prime}(1, \chi)$ was sought for and the following is of fundamental relevance.

$$
L^{\prime}(1, \chi)=-L(1, \chi) \log q+\frac{1}{2 q} \sum_{\mu=1}^{q-1} \chi(\mu) R^{\prime}\left(\frac{\mu}{q}\right) .
$$


As an analogy to [HKT, Theorem] ([Vista, Chapter 8]), we shall prove the following result.

Theorem 2. The finite expression

$$
L^{\prime}(1, \chi)=(\gamma+\log 2 \pi) L(1, \chi)+ \begin{cases}\frac{1}{q} \sum_{\nu=1}^{q-1} R\left(\frac{\nu}{q}\right) G(\nu, \chi), & \chi \text { even } \\ \frac{\pi i}{q} \sum_{\nu=1}^{q-1} \log \Gamma\left(\frac{\nu}{q}\right) G(\nu, \chi), & \chi \text { odd }\end{cases}
$$

is equivalent to $(2.2)$.

\section{Proof}

To prove Theorem 2, we use many results which are obtained in [HKT] ([Vista, Chapter 8]), which we state as a lemma.

\section{Lemma 3.}

([Vista, (8.28)])

$$
\sum_{a=1}^{q-1} \chi(a) \psi\left(\frac{a}{q}\right)=-q L(1, \chi)
$$

([Vista, (8.39-1)])

$$
\sum_{a=1}^{q-1} \chi(a) \cos \left(\frac{2 \pi a \mu}{q}\right)=\frac{1+\chi(-1)}{2} G(\mu, \chi)
$$

and

([Vista, (8.39-2)] $\quad \sum_{a=1}^{q-1} \chi(a) \sin \left(\frac{2 \pi a \mu}{q}\right)=\frac{1-\chi(-1)}{2 i} G(\mu, \chi)$.

We also recall the orthogonality relation among the characters.

Lemma 4. Let $\chi$ denote a Dirichlet character $\bmod q, q \geq 3$. Then

$$
\sum_{\chi \text { even }} \chi(n)=\left\{\begin{array}{llll}
0 & \text { if } & n \neq \equiv \pm 1 & (\bmod q) \\
\frac{\varphi(q)}{2} & \text { if } & n \equiv \pm 1 & (\bmod q) .
\end{array}\right.
$$


and

$$
\sum_{\text {odd }} \chi(n)=\left\{\begin{array}{ccc}
0 & \text { if } & n \neq \equiv 1 \quad(\bmod q), \\
\frac{\varphi(q)}{2} & \text { if } & n \equiv 1 \quad(\bmod q), \\
-\frac{\varphi(q)}{2} & \text { if } & n \equiv-1 \quad(\bmod q),
\end{array}\right.
$$

where the sum is extended over all even and odd characters, respectively.

\section{Proof of Theorem 2}

It could be immediately seen that (2.2) implies (2.12). Indeed, substituting (2.2) in $\sum_{a=1}^{q-1} \chi(a) R^{\prime}\left(\frac{a}{q}\right)$ in (2.11) and using Lemma 4, we find that

$$
\begin{aligned}
& L^{\prime}(1, \chi)=-L(1, \chi) \log q-\frac{1}{q}(\gamma+\log 2 \pi q) \sum_{a=1}^{q-1} \chi(a) \psi\left(\frac{a}{q}\right) \\
& +\frac{1}{q} \sum_{\mu=1}^{q-1} R\left(\frac{\mu}{q}\right) \sum_{a=1}^{q-1} \chi(a) \cos \frac{2 \pi \mu a}{q}-\frac{\pi}{q} \sum_{\mu=1}^{q-1} \log \Gamma\left(\frac{\mu}{q}\right) \sum_{a=1}^{q-1} \chi(a) \sin \frac{2 \pi \mu a}{q} .
\end{aligned}
$$

Now substituting from Lemma 3 and classifying the parity of $\chi$, we conclude (2.12).

To prove the converse, we consider for $(p, q)=1$

$$
S(p)=\sum_{\chi \neq \chi_{0}} \chi\left(p^{-1}\right) L^{\prime}(1, \chi) .
$$

Now substituting (2.11), we obtain

$$
S(p)=-(\log q) \sum_{\chi \neq \chi_{0}} \chi\left(p^{-1}\right) L(1, \chi)+\frac{1}{2 q} \sum_{a=1}^{q-1} R^{\prime}\left(\frac{a}{q}\right) \sum_{\chi \neq \chi_{0}} \chi\left(p^{-1}\right) \chi(a) .
$$

We rewrite the inner sum of the second term on the right as

$$
\sum_{\chi} \chi\left(p^{-1}\right) \chi(a)-\chi_{0}(a)
$$

and using Lemma 4, we deduce that

(3.3) $S(p)=-(\log q) \sum_{\chi \neq \chi_{0}} \chi\left(p^{-1}\right) L(1, \chi)+\frac{\varphi(q)}{2 q} R^{\prime}\left(\frac{p}{q}\right)-\frac{1}{2 q} \sum^{*} R^{\prime}\left(\frac{a}{q}\right)$, 
where $*$ on the summation sign means that the sum is over integers $1 \leq a \leq$ $q-1$ relatively prime to $q$.

Further from (3.3) we obtain

$$
\frac{\varphi(q)}{2 q} R^{\prime}\left(\frac{p}{q}\right)=S(p)+(\log q) \sum_{\chi \neq \chi_{0}} \chi\left(p^{-1}\right) L(1, \chi)+\frac{1}{2 q} \sum^{*} R^{\prime}\left(\frac{a}{q}\right)
$$

To transform each term on the right of (3.4), we show

Lemma 5. We have the following.

$$
\sum^{*} R^{\prime}\left(\frac{a}{q}\right)=-\gamma_{1} \varphi(q)+q \sum_{d \mid q} \frac{\mu(d)}{d} \log ^{2} \frac{q}{d}+2 \gamma q \sum_{d \mid q} \frac{\mu(d)}{d} \log \frac{q}{d}
$$

$$
\begin{aligned}
& S(p)\left(=\sum_{\chi \neq \chi_{0}} \chi\left(p^{-1}\right) L^{\prime}(1, \chi)\right) \\
& =(\gamma+\log 2 \pi) \sum_{\chi \neq \chi_{0}} \chi\left(p^{-1}\right) L(1, \chi)+\frac{\varphi(q)}{q} \sum_{\nu=1}^{q-1} R\left(\frac{\nu}{q}\right) \cos \frac{2 \pi \nu p}{q} \\
& -\sum_{d \mid q} \frac{\mu(d)}{d}\left(\frac{1}{2} \log ^{2} d+(\log 2 \pi)(\log d)\right)-\pi \frac{\varphi(q)}{q} \sum_{\nu=1}^{q-1} \log \Gamma\left(\frac{\nu}{q}\right) \sin \frac{2 \pi \nu p}{q}
\end{aligned}
$$

and

([Vista, (8.46)])

$$
\sum_{\chi \neq \chi_{0}} \chi\left(p^{-1}\right) L(1, \chi)=\frac{\varphi(q)}{q}\left(-\psi\left(\frac{p}{q}\right)-\gamma-\log q\right)+\sum_{d \mid q} \frac{\mu(d)}{d} \log d .
$$

Proof. By (2.5), we find that

$$
\sum^{*} R^{\prime}\left(\frac{a}{q}\right)=\sum_{d \mid q} \mu(d) \sum_{d \mid a} R^{\prime}\left(\frac{a}{q}\right)=\sum_{d \mid q} \mu(d) \sum_{a=1}^{\frac{q}{d}-1} R^{\prime}\left(\frac{a}{\frac{q}{d}}\right) .
$$

Now substituting (2.4), we conclude (3.5). Here we have used the fact that used $R^{\prime}(1)=-\gamma_{1}$.

To prove (3.6), we substitute (2.12) and obtain

$$
S(p)=(\gamma+\log 2 \pi) \sum_{\chi \neq \chi_{0}} \chi\left(p^{-1}\right) L(1, \chi)+\frac{1}{q} S^{\text {even }}(p)+\frac{1}{q} S^{\text {odd }}(p),
$$


where

$$
S^{\text {even }}(p)=\sum_{\substack{\chi \neq \chi_{0} \\ \chi \text { even }}} \chi\left(p^{-1}\right) \sum_{\nu=1}^{q-1} R\left(\frac{\nu}{q}\right) G(\nu, \chi)
$$

and

$$
S^{\text {odd }}(p)=\sum_{\chi \text { odd }} \chi\left(p^{-1}\right) \pi i \sum_{\nu=1}^{q-1} \log \Gamma\left(\frac{\nu}{q}\right) G(\nu, \chi) .
$$

Now by Lemma 3 we have

$$
S^{\text {odd }}(p)=-\pi \varphi(q) \sum_{\nu=1}^{q-1} \log \Gamma\left(\frac{\nu}{q}\right) \sin \frac{2 \pi \nu p}{q} .
$$

We write

$$
\begin{aligned}
S^{\text {even }}(p) & =\sum_{\nu=1}^{q-1} R\left(\frac{\nu}{q}\right) \sum_{\chi \text { even }} \chi\left(p^{-1}\right) G(\nu, \chi)-\sum_{\nu=1}^{q-1} R\left(\frac{\nu}{q}\right) G\left(\nu, \chi_{0}\right) \\
& =S_{1}(p)-S_{2}(p)
\end{aligned}
$$

(say). Then using Lemma 3 one has

$$
S_{1}(p)=\varphi(q) \sum_{\nu=1}^{q-1} R\left(\frac{\nu}{q}\right) \cos \frac{2 \pi \nu p}{q} .
$$

On the other hand, using (2.8) and (2.10), we deduce that

$$
S_{2}(p)=\sum_{\nu=1}^{q-1} R\left(\frac{\nu}{q}\right) \sum_{d \mid(q, \nu)} d \mu\left(\frac{q}{d}\right)=\sum_{d \mid q} d \mu\left(\frac{q}{d}\right) \sum_{\mu=1}^{\frac{q}{d}-1} R\left(\frac{\mu}{\frac{q}{d}}\right) .
$$

Substituting (2.3), we deduce that

$$
S_{2}(p)=\sum_{d \mid q} \frac{q}{d} \mu(d)\left(\frac{1}{2} \log ^{2} d+(\log 2 \pi)(\log d)\right) .
$$

Further substituting (3.11) and (3.12) in (3.10), we conclude that

$S^{\text {even }}(p)=\varphi(q) \sum_{\nu=1}^{q-1} R\left(\frac{\nu}{q}\right) \cos \frac{2 \pi \nu p}{q}-\sum_{d \mid q} \frac{q}{d} \mu(d)\left(\frac{1}{2} \log ^{2} d+(\log 2 \pi)(\log d)\right)$

Finally substituting (3.9) and (3.13) into (3.7), we conclude (3.6). This completes the proof of Lemma 5. 
We are now in a position to complete the proof of Theorem 2. Substituting (3.5) and (3.6) in (3.4),

$$
\begin{aligned}
& \frac{\varphi(q)}{2 q} R^{\prime}\left(\frac{p}{q}\right)=(\gamma+\log 2 \pi q) \sum_{\chi \neq \chi_{0}} \chi\left(p^{-1}\right) L(1, \chi)+\frac{\varphi(q)}{q} \sum_{\nu=1}^{q-1} R\left(\frac{\nu}{q}\right) \cos \frac{2 \pi \nu p}{q} \\
& -(\log 2 \pi) \sum_{d \mid q} \frac{\mu(d)}{d} \log d-\pi \frac{\varphi(q)}{q} \sum_{\nu=1}^{q-1} \log \Gamma\left(\frac{\nu}{q}\right) \sin \frac{2 \pi \nu p}{q} \\
& -\frac{\varphi(q)}{2 q} \gamma_{1}+\frac{1}{2} \sum_{d \mid q} \frac{\mu(d)}{d}\left(\log ^{2} q-2(\log q)(\log d)\right)+\gamma \sum_{d \mid q} \frac{\mu(d)}{d}(\log q-\log d)
\end{aligned}
$$

since the terms $\sum_{d \mid q} \frac{\mu(d)}{d} \log ^{2} d$ cancel.

Finally, substituting the last formula in Lemma 5 and noting that all the terms $\sum_{d \mid q} \frac{\mu(d)}{d} \log d$ cancel, we are left with

$$
\begin{aligned}
& R^{\prime}\left(\frac{p}{q}\right)=2\left(-\psi\left(\frac{p}{q}\right)-\gamma-\log q\right)(\gamma+\log 2 \pi q)+2 \sum_{\nu=1}^{q-1} R\left(\frac{\nu}{q}\right) \cos \frac{2 \pi \nu p}{q} \\
& -2 \pi \sum_{\nu=1}^{q-1} \log \Gamma\left(\frac{\nu}{q}\right) \sin \frac{2 \pi \nu p}{q}-\gamma_{1}+\frac{\log ^{2} q}{\varphi(q)} \sum_{d \mid q} \frac{q \mu(d)}{d}+\frac{\gamma \log q}{\varphi(q)} \sum_{d \mid q} \frac{q \mu(d)}{d}
\end{aligned}
$$

which is (2.2) in view of (2.6). This complete the proof of Theorem 2.

Acknoeledgement The authors would like to thank Professor Tanigawa for many enlightening discussions and useful comments.

\section{References}

[Ap] T. M. Apostol, Introduction to analytic number theory, Springer Verag, Berlin etc. 1976.

[CSel] S. Chowla and A. Selberg, On Epstein's zeta-function (I), Proc. Nat. Acad. Sci. USA 35 (1949), 371-374; Collected Papers of Atle Selberg I, Springer Verlag, 1989, 367-370. The Collected Papers of Sarvadaman Chowla II, CRM, 1999, 719-722.

[Den] C. Deninger, On the analogue of Chowla-Selberg formula for real quadratic fields, J. Reine Angew. Math. 351 (1984), 171-191. 
[Erd] A. Erdélyi (ed), W. Magnus, F. Oberhettinger and F. G. Tricomi, Higher transcendental functions, vol I, McGraw-Hill Book Company, Inc., New York-Toronto-London, 1953.

[Gu] M. Gut, Die Zetafunktion, die Klassenzahl und die Kronecker'sche Grenzformel eines beliebigen Kreiskörpers, Comment. Math. Helv. 1 (1929), 160-226.

[HKT] M. Hashimoto, S. Kanemitsu and M. Toda, On Gauss' formula for and finite expressions for the L-series at 1, J. Math. Soc. Japan, 60 (2008), 219-236.

[Vista] S. Kanemitsu and H. Tsukada, Vistas of special functions, World Scientific, New Jersey-London-Singapore etc., 2007.

[Klu] D. Klusch, On the Taylor expansion of the Lerch zeta-function, $J$. Math. Anal. Appl. 170 (1992), 513-523.

[Kum] H. Kumagai, On unified Kronecker limit formulae, Kyushu J. Math. 56 (2002), 41-51.

[Kur] N. Kurokawa, One hundred years of zeta regularized product. The 39th Algebra Sympos. 1994, 153-166.

[LG] A. Laurinchikas and R. Garunkstis, The Lerch zeta-function, Kluwer Academic Publ., Dordrecht-Boston-London 2002.

[Lan] E. Landau, Über die zu einem algebraischen Zahlkörper gehörige Zetafunction und die Ausdehnung der Tscheschefschen Primzahltheorie auf das Problem der Primiideal, J. Reine Angew. Math. 125 (1903), 176188=Collected Papers Vol. 1, pp. 201-325.

[Ler1] M. Lerch, Note sur la function $K(w, x, s)=\sum \frac{e^{2 \pi k i w}}{(w+k)^{s}}$ Acta Math. 11 (1887), 19-24.

[Ler2] M. Lerch, Sur quelques formules du nombre des classes, Bull. Sci. Math. 21 (1897), 290-304.

[LCK] H. -L. Li, K. Chakraborty and S. Kanemitsu, On the values of a class of Dirichlet series at rational arguments, to appear.

[Lip] R. Lipschitz, Untersuchungen der Eigenschaften einter Gattung von unendlichen Reihen, J. Reine Angew. Math. 105 (1889), 127-156. 
[SelC] A. Selberg and S.Chowla, On Epstein's zeta-function, J. Reine Angew, Math. 227 (1967), 86-110; Collected Papers of Atle Selberg I, Springer Verlag, 1989, 521-545; The Collected Papers of Sarvadaman Chowla II, CRM, 1999, 1101-1125.

[SC] H. M. Srivastava and J.-S. Choi, Series Associated with the zeta and related functions, Kluwer Academic Publ., Dordrecht 2001.

[Wil1] J. R. Wilton, A proof of Burnside's formula for $\log \Gamma(x+1)$ and certain allied properties of Riemann's $\zeta$-function, Messenger Math. 52 (1922/1923), 90-93.

Addresses of the authors

K. Chakraborty

Harish-Chandra Research Institute

Chhatnag Road, Jhusi,

Allahabad 211 019, India

E-mail:kalyan@hri.res.in

S. Kanemitsu

Graduate School of Advanced Technology

Kinki University

Iizuka, Fukuoka 820-8555, Japan

E-mail: kanemitu@fuk.kindai.ac.jp

Takako Kuzumaki

Department of Mathematical Design

Gifu University

Gifu, 501-1193, Japan

E-mail: kuzumaki@math.gifu-u.ac.jp

Received on March 20, 2009

Accepted on March 21, 2009 